\title{
Master Formula of Pharmaceutical Product
}

National Cancer Institute

\section{Source}

National Cancer Institute. Master Formula of Pharmaceutical Product. NCI Thesaurus.

Code C42668.

A document or set of documents specifying the starting materials with their quantities and the packaging materials, together with a description of the procedures and precautions required to produce a specified quantity of a finished pharmaceutical product as well as the processing instructions, including in-process controls. 